\title{
Magnetic and Dyed Microcapsules From the Spores of Clubmoss
}

\author{
Wen Cai ${ }^{\dagger}$, Charles Bradbury, Stephen Cowling and John Goodby *t \\ Department of Chemistry, University of York, York, United Kingdom
}

In this article we describe the preparation of bioavailable microcapsules derived from spores of clubmoss (Lycopodium clavatum) that are composed of protein-free, nonallergenic, and porous sporopollenin, often described as nature's most robust polymeric system. We describe the adsorption of magnetic materials onto the exterior surfaces of the empty microcapsules and the incorporation of molecular passengers, thereby producing stable, monodispersed, bifunctional particles/vehicles of 25 microns in size that can respond to, or targeted by, applied fields.

Keywords: microcapsules, spores, sporopollenin, nanomagents, dyes

\section{INTRODUCTION}

\section{OPEN ACCESS}

Edited by:

Mamatha Nagaraj,

University of Leeds, United Kingdom

Reviewed by:

Amir Maldonado,

Universidad de Sonora, Mexico

Amiya Kumar Panda,

Vidyasagar University, India

*Correspondence:

John Goodby

john.goodby@york.ac.uk

${ }^{\dagger}$ These authors share first authorship

Specialty section:

This article was submitted to

Soft Matter Physics,

a section of the journal

Frontiers in Physics

Received: 13 December 2021

Accepted: 31 January 2022

Published: 23 February 2022

Citation:

Cai W, Bradbury C, Cowling $S$ and Goodby J (2022) Magnetic and Dyed

Microcapsules From the Spores

of Clubmoss.

Front. Phys. 10:834455.

doi: 10.3389/fphy.2022.834455
Microcapsules and functional particles have a wide variety of applications, from paints, coatings, E-inks, thermochromics, time-release pharmaceuticals, to visual indicators, etc. The shells of synthetic particles and microcapsules are usually composed of lipids or polymers, or materials such as alginates, gelatin and gum Arabic. Their sizes and stabilities vary depending on material selection, production, and the environment of their use. Furthermore, it has recently been noted that certain polymeric microcapsules are detrimental to the environment. Comparatively, nature provides its own variety of microcapsules and particles based on cell walls and membranes, but unlike their synthetic equivalents, they possess discrete size, shape, morphology and uniform (mono) dispersity within each species.

Plant, fungal and bacterial spores and plant pollen provide a unique and extremely large collection of biological particles and microcapsules that range in size from a few microns up to millimetres depending on genus [1-3]. Their shapes and sizes are genus specific and reproducible, and range from spherical to oblate and prolate. The inner layer of the cell walls of spores and pollen, called the intine, consists mainly of cellulose and pectin [4], which degrades during fossilization. Their outer shells, called exines, are composed of sporopollenin, which is often described as nature's most robust polymeric system [5]. The walls are usually composed of a $\mathrm{N}$-free polymeric substance related to terpenes, with chemical formulae approximating $\mathrm{C}_{90}, \mathrm{H}_{130-158}, \mathrm{O}_{24-44}$. Although the surface of pollen grains and spores from various species may appear different under the scanning electron microscope (SEM), transmission electron microscopy (TEM) demonstrates that the layers present within their walls are broadly similar [6]. Sporopollenin is chemically unsaturated and is corroded by oxygen, but is otherwise resistant even to strong alkaline substances and organic acids [3, 7]. Thus, in the plant world Sporopollenin is one of the most resistant substances, and consequently, pollen grains and spores can be preserved under anoxic conditions in lakes and fens for thousands to millions of years [8]. They can therefore be used for the reconstruction of earlier vegetation- and climate-conditions.

When the biological content (protoplast) of spores and pollen is removed, microcapsules (MC) are generated that are protein-free, non-allergenic, and porous. As the outer shells of the resulting microcapsules are composed of a highly conjugated organic polymer possessing $p$-coumaric and ferulic acid moieties, with both phenolic and acid groups located on its surface, the contents of an 


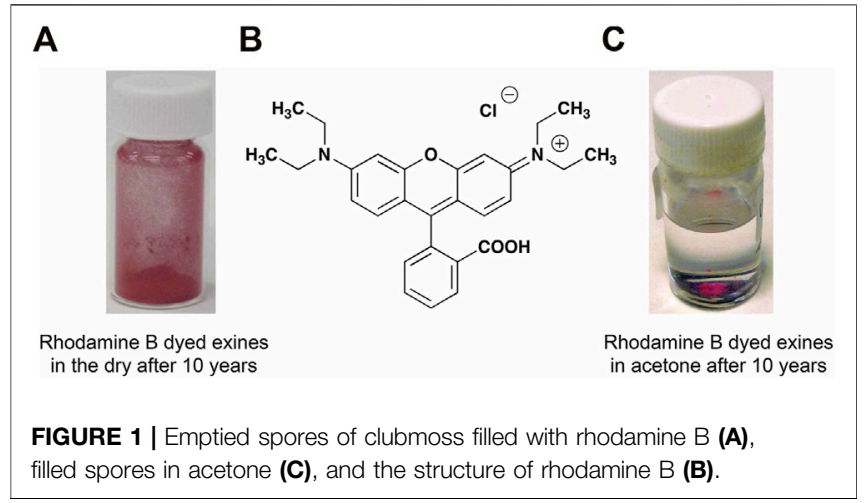

adsorbed guest material, will be protected from oxidation via preceding reactions with oxygen, oxygen radicals or the air where scavenging by sporopollenin will take place. For example, we have demonstrated that the laser dye rhodamine $\mathrm{B}$ remains stable within microcapsules of clubmoss (Lycopodium clavatum) for a number of years, as shown in Figure 1.

All of these properties are not usually available from synthetic equivalents. Early applications of such natural microcapsules have been limited to the encapsulation of materials that are relevant in health-care, such as pharmaceuticals, drug delivery [9-11], food additives [12], mRNA isolation [13], and MRI contrast agents [14]. However, spores and pollen have found uses as microcapsules for functional materials, and therefore their possible futures in applications still remain an open and exciting area for exploration, particularly in novel areas of transporting and vectoring more than one functional material, protected by biological microcapsules, to molecular or macroscopic targets.

In this article we demonstrate the incorporation of a dye into microcapsules derived from clubmoss (Lycopodium clavatum), and then with the impregnation of magnetic particles $\left(\mathrm{Fe}_{3} \mathrm{O}_{4}\right.$ or $\gamma-\mathrm{Fe}_{2} \mathrm{O}_{3}$ ) we produce coloured particles that can be moved with the application of a magnetic field.

\section{MATERIALS AND METHODS \\ Processing and Preparation of Microcapsules}

Removal of the biologically active cores of spores and pollen is genus specific, and methodologies have not yet been developed for all of the species that might be of interest in various applications. As a starting point, we used a methodology for processing clubmoss that was derived from literature reports by Zetzsche et al [15] and Atkin et al [16]. A method we adapted from a patent by Atkin et al. [17] enabled the recovery of pure, sporopollenin exospore shells, leaving only empty microcapsules, free of any potential allergens. It should be noted that the methods of Zetzsche and Atkin can result in partial rupturing of the resultant microcapsules, $(2-5 \%$ when averaged over several SEM studies of 20-25 spores), see Figure 2 (left), whereas our numerous experimental procedures, made on an ad-hoc basis, identified a suitable methodology that could eliminate this problem and maintain the integrity of the microcapsules, as

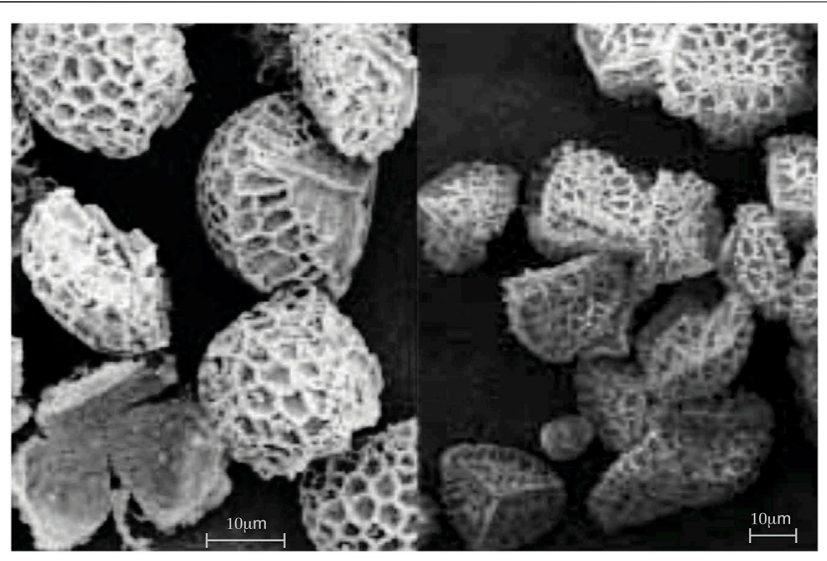

FIGURE 2 | Exines produced from Lycopodium clavatum. Left image shows SEMs of exines where some are fractured, and right, processed spores where the exines are not broken.

shown in Figure 2 (right). Over various studies using the bulk quantity of exines, and studies of new batches our average count of ruptured exines was between 2 and $5 \%$.

Our later attempts at processing clubmoss were based on a US patent [17]. However, it was found that severe degradation occurred during the acid treatment stage of the programme (2 $\times 5$ days stirring under reflux in $85 \%$ orthophosphoric acid). The brown product was examined under SEM and found to consist of macaroni-like material. This was not only attributed to the severe acid treatment conditions, but also to the type of Clubmoss used, i.e., Aldrich Cat. No. 19108. This material, when examined by SEM, showed very smooth-surfaced tetrahedra, presumably very prone to attack by reagents. Another commercial source for the spores was identified as G Baldwin \& Co., London.

The procedure eventually developed was based on the patent [17], example 13, with slight modifications, up to the point of isolation of the unbleached "base hydrolysed" or "BHS" exine shells. It was found that the bleaching conditions specified in the examples were too aggressive $\left(60^{\circ} \mathrm{C}\right)$ and the sporopollenin product obtained was found to be cracked (SEM) as shown in Figure 2, i.e., oxidatively degraded. However, milder treatment with bleach $\left(35^{\circ} \mathrm{C}\right)$ gave good quality sporopollenin and exines, see Figure 3, which were employed in these studies, and in comparison the figure to the right that shows the raw spores with the cytoplasm still inside the exine.

\section{Experimental Methods for the Preparation of Exines of Lycopodium clavatum}

Firstly, it should be noted that sporopollenin has been used as a cheap flammable powder for pyrotechnics. Therefore, appropriate precautions should be taken as described in the Apparatus section below. Regular safety rules for operations in organic chemistry laboratories, such as handing of materials inside the fume cupboards in the absence of sources of ignition should be made. 

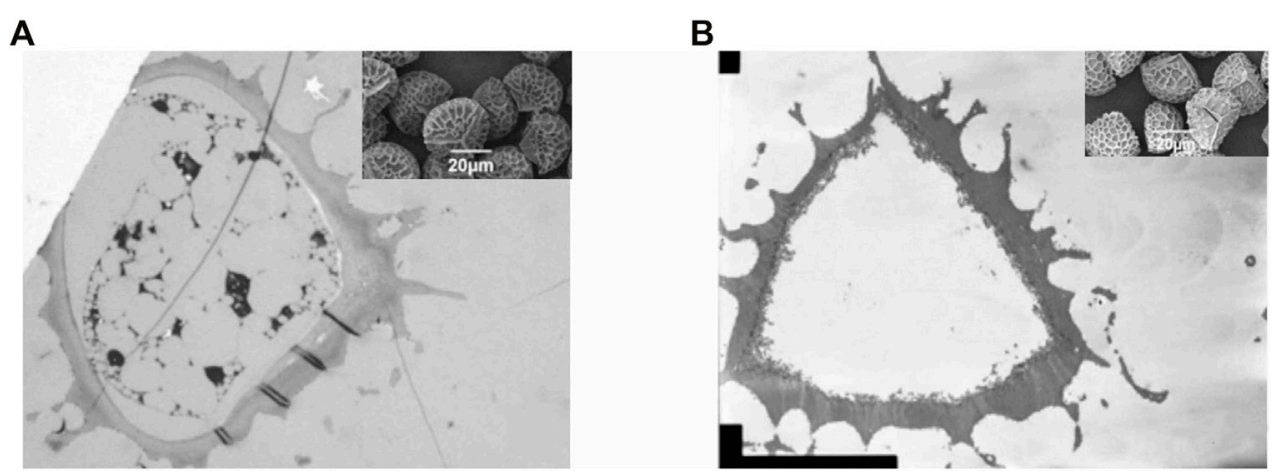

FIGURE 3 | (A), an SEM of clubmoss (Lycopodium clavatum) before removal of the biologically active core, and (B), the exine shell of the spore.

Apparatus: In all stages, a 2 L 3-necked round bottom flask was employed, using a (large) magnetic stirrer with metal-bath heating. A thermometer and a water condenser were used. No inert gas was used, other than to blanket the vessel atmosphere when powder was manipulated (danger of spark generation due to static, and therefore of fire in the presence of flammable solvent). All operations were carried out in a fume cupboard, and long gloves were worn for most operations.

Stage 1: "G Baldwin spores" ( $250 \mathrm{~g}$ ) was carefully added to a plastic container and, under an Argon atmosphere, acetone (1 L) was added, by slow introduction down the side of the container. The container was closed, shaken and the slurry obtained was then poured via a funnel into the RB flask. The acetone-wet solids were washed into the flask with a further quantity of acetone (300 ml-Note 1). The mixture was stirred and heated under reflux (pot temp. ca. $60^{\circ} \mathrm{C}$ ) for $4 \mathrm{~h}$. The mixture was then cooled to $40^{\circ} \mathrm{C}$ and the spores filtered through a glass sinter, washed with acetone $(300 \mathrm{ml})$ and dried in air to constant weight. The weight loss recorded was $4.4 \%$.

Note 1: The volume of acetone was increased from that specified in the Patent, in order to achieve good stirring.

Stage 2: The Stage 1 product (239 g) was charged under Argon atmosphere to the flask and $6 \%$ aqueous $\mathrm{KOH}$ solution $(51 \mathrm{~g}$ as $100 \% \mathrm{KOH}$, dissolved in water to a total volume of $850 \mathrm{ml}$ ) added. Manual swirling made commencement of stirring by the magnet an easier process. The stirred mixture was heated to reflux (pot temperature ca. $100^{\circ} \mathrm{C}$ ) for $6 \mathrm{~h}$. The slurry was allowed to cool, with continued stirring, overnight. The resulting brown syrup was filtered in four batches. It was found necessary to add hot $\left(80^{\circ} \mathrm{C}\right)$ water $(2$ vols $)$ to each filtration batch, in order to attain reasonable filtration rates. Each cake was slurred with water (at $80^{\circ} \mathrm{C}, 5 \times 400 \mathrm{ml}$ ) and finally washed with hot water (displacement, $500 \mathrm{ml}$, i.e., total water washings $12.5 \mathrm{~L}$ ). Initially, the filtrate/washings were dark brown in colour, but eventually these became colourless. The resulting orange-brown cake was pulled as dry as possible and then dried in air o/n.

Stage 3: The damp cake from Stage $2(280 \mathrm{~g})$ was suspended in $6 \%$ aqueous $\mathrm{KOH}(750 \mathrm{ml})$, allowance being made for the water content of the cake (estimated at $80 \mathrm{~g}$ ).

The Stage 2 procedure was repeated exactly, except that the cake was washed, not only with hot water (total $8 \mathrm{~L}$, all in one filtration batch), but also sequentially with EtOH $(2 \times 500 \mathrm{ml}$ at $\left.60^{\circ} \mathrm{C}\right)$, water $\left(2 \times 500 \mathrm{ml}\right.$ at $\left.80^{\circ} \mathrm{C}\right)$ and finally with warm EtOH $(2$ $\times 500 \mathrm{ml}$ ).

Stage 4: The Stage 3 EtOH-damp cake ( $300 \mathrm{~g})$ was charged to the flask under Argon and EtOH $(650 \mathrm{ml})$ added, allowance having been made for the EtOH content of the cake-ca. $100 \mathrm{~g}$ ). The mixture was heated to reflux with stirring and this condition maintained for $2 \mathrm{~h}$ After cooling to ca. $40^{\circ} \mathrm{C}$, the brownish-yellow product was isolated by filtration (in one easy operation) and slurry-washed with EtOH $(2 \times 500 \mathrm{ml})$ and then with DCM $(2 \times 500 \mathrm{ml})$. The cake was pulled as dry as possible.

Stage 5: The damp cake from Stage 4 was charged to the flask and DCM $(600 \mathrm{ml})$ added (allowance having been made for ca. $150 \mathrm{ml}$ of DCM in the cake). The slurry was heated under reflux, with stirring, for $2 \mathrm{~h}$. After cooling to RT, the product was filtered, washed with DCM $(250 \mathrm{ml})$ and dried in air o/n. (This stage was added in order to ensure thorough removal of fatty material-also avoidance of acetone was considered important because of possible hazard in reaction of residual solvent with bleach in the next stage).

Stage 6: The "exine BHS shells" were suspended in 7\% sodium hypochlorite solution (50 v/w, Note 2) and the slurry warmed with stirring to $35^{\circ} \mathrm{C}$. This condition was maintained for $2 \mathrm{~h}$ (or until the colour of the solids became almost white, i.e., very pale cream-a white product is obtainable by further reaction times or higher temperatures, but oxidative degradation is noted). The slurry was then cooled to RT and immediately filtered and washed with water until a negative starch iodide test was obtained. Washing was next effected with $\mathrm{EtOH}(400 \mathrm{ml})$ and then with acetone $(400 \mathrm{ml})$ and finally the product was dried in vacuo over $\mathrm{P}_{2} \mathrm{O}_{5}$. Yield of bleached spores $=73.3 \mathrm{~g}(29.3 \%$ on input $)$.

Note 2: A new $500 \mathrm{ml}$ bottle of sodium hypochlorite (13\% active chlorine) was purchased from Acros Organics/Fisher Scientific (or elsewhere) immediately prior to practical work on the last stage and was stored in the lab fridge-dilution to $7 \%$ active with water was carried out before each experiment $b$ ) a Certificate of Analysis was obtained from the bleach supplier and c) small (1 g) bleaching trials were carried out in order to confirm the appropriate temperature and stir time necessary to achieve a good final product colour, without inflicting oxidative degradation. This could only be achieved by trial and error 
TABLE 1 | Experimental conditions for the adsorption of rhodamine B (rows one and 2) and naphthol blue-black (row 3): pre-treatment solvent, elution solvent (c. $0.1 \mathrm{~g} \mathrm{~L}^{-1}$ ), and volume and quantity of exines used in the 'column'.

\begin{tabular}{|c|c|c|c|c|}
\hline Pretreatment solvent & $\begin{array}{c}\text { Vol of pre-treatment solvent } \\
\text { used }(\mathrm{ml})\end{array}$ & Dye solvent & Volume (ml) & $\begin{array}{l}\text { Wt of exine used } \\
\text { (g) }\end{array}$ \\
\hline $5 \mathrm{M} \mathrm{HCl}$ & 3 & Ethanol & 2 & 0.2 \\
\hline $5 \mathrm{M} \mathrm{HCl}$ & 3 & Acetone & 2 & 0.2 \\
\hline Ethanol & 1 & Acetone & 2 & 0.2 \\
\hline
\end{tabular}

TABLE 2 | Details of dye solvent used concentration, volume added to column and weight of exine.

\begin{tabular}{lccc} 
Solvent & Dye conc. $\left(\mathbf{g L}^{-\mathbf{1}}\right)$ & Vol $(\mathbf{m l})$ & Wt of exine $\mathbf{( g )}$ \\
\hline Deionized water & 0.1 & 2 & 0.2 \\
Acetone & 0.1 & 2 & 0.2 \\
Ethanol & 0.1 & 2 & 0.2 \\
Dichloromethane & 0.1 & 2 & 0.2 \\
Water/acetone (1:1) & 0.1 & 2 & 0.2 \\
Acetonitrile & 0.1 & 1 & 0.1 \\
Chloroform & 0.1 & 1 & 0.1 \\
Diethyl phthalate & 0.1 & 1 & 0.1 \\
Ethanediol & 0.1 & 2 & 0.2 \\
2-propanol & 0.1 & 2 & 0.2 \\
\hline
\end{tabular}

with SEM being used to test the quality of the product sporopollenin.

\section{Preparation of Functionalized Microcapsules Magnetic Microcapsules (MS1) Prepared via Nanoparticles Synthesized Within Bleached Emptied Spores (BEICs)}

A solution was prepared by mixing $\mathrm{FeCl}_{3}$ (Aldrich, UK, $8.11 \mathrm{~g}$; $0.05 \mathrm{~mol}$ ), $\mathrm{FeCl}_{2} \cdot \mathrm{H}_{2} \mathrm{O}$ (20.27g; $\left.0.14 \mathrm{~mol}\right), \mathrm{HCl}(5 \mathrm{M}, 5 \mathrm{ml})$, deionized water $(40 \mathrm{ml})$ and ethanol $(5 \mathrm{ml})$. The mixture was heated to $40^{\circ} \mathrm{C}$ until all of the salts dissolved. BEICs $(0.2 \mathrm{~g})$ were placed under a vacuum for $10 \mathrm{~min}$, and then they were added to $15 \mathrm{ml}$ of the prepared solution and stirred for $2 \mathrm{~h}$ at RT. Subsequently, the iron-salt-BEICs admixtures were filtered off and washed very quickly with water $(10 \mathrm{ml})$. Then they were placed in an ammonia solution (water:ammonia $=96: 6,100 \mathrm{ml}$ ) and stirred for $1 \mathrm{~h}$. The product was filtered off and washed with water $(100 \mathrm{ml})$. Finally, the product was dried under vacuum.

\section{Magnetic Microcapsules (MS2) Produced by Doping Nanoparticles Onto BEICs Using the "Column" Method}

Magnetic nanoparticles $\left(\gamma-\mathrm{Fe}_{2} \mathrm{O}_{3}, 0.0193 \mathrm{~g}\right)$ were dispersed in water $5 \mathrm{ml}$ in a sample vial using ultrasound for $1 \mathrm{~h}$ at $30^{\circ} \mathrm{C}$. Meanwhile, BEICs $(0.1003 \mathrm{~g})$ were packed in a mini "column". The magnetic nanoparticle fluid mixture $(2 \mathrm{ml})$ was dropped onto the "column" and forced to progress down the column under vacuum. Then the magnetic nanoparticle fluid mixture $(2 \mathrm{ml})$ was added to the "column" again and allowed to flow through it for overnight without any extra treatment. Finally, the “column" was washed with acetone $(2 \mathrm{ml})$ and treated under vacuum until the product dried.

\section{Magnetic Microcapsules Produced by Doping Nanoparticles Onto BEICs Using Ultrasonic Bath}

Magnetic nanoparticles $\left(\gamma-\mathrm{Fe}_{2} \mathrm{O}_{3}\right.$, half spatula) were dispersed in water $(5 \mathrm{ml})$ by ultrasound at $50^{\circ} \mathrm{C}$. The BEICs $(0.1124 \mathrm{~g})$ were added to the fluid and treated with ultrasound at $50^{\circ} \mathrm{C}$ for $2 \mathrm{~h}$. Then the filled BEICs were washed with acetone and re-dispersed in acetone $(5 \mathrm{ml})$ using ultrasound for $10 \mathrm{~min}$. Finally, they were filtered and dried in air overnight.

The stabilities of the nanoparticles within the emptied spores were thought to depend mostly on two possibilities, 1) the nature of the syntheses of the nanoparticles by either bottom-up or top-down methodologies, and/or 2) the adsorption of an addlayer around the particle surfaces relative to the intine layer. The first will be affected by particle growth, whereby defect formation and surface energy can affect size, the second by adsorption of any surfactants that might remain within the exine shells.

\section{Dye Adsorption Into Emptied Spores}

Prior to the studies on dye adsorption into emptied exines, experimental conditions were evaluated for determining the best method of column packing and reproducibility. A "column" using empty exines was set up in a Pasteur pipette to provide a point of reference for the study. The neck of the pipette was plugged with glass wool, and a weighed quantity of the exines was added, followed by a layer of sand. The sand layer ensured minimum disturbance to the spores layer while the dye solution was added. The following conditions were then used to examine the most efficient and best arrangement of packing.

1) The column was packed and used without treatment.

2) The column was packed and then compacted using a weak vacuum.

3) The exines were subjected to a vacuum and left in air for $3 \mathrm{~h}$, then packed into the column.

4) The spores were subjected to a vacuum, packed into a column and then compacted using a vacuum.

Using method 4, the packed empty exine columns were pretreated by sucking a volume of $\mathrm{HCl}$, ethanol or dichloromethane into the column. Subsequently, a $2 \mathrm{ml}$ solution $\left(1 \mathrm{~g} \mathrm{~L}^{-1}\right)$ of a dye in a solvent was loaded onto the column and sucked through using a vacuum. The filtrate was isolated and used to determine how much dye had adsorbed onto the "column". 

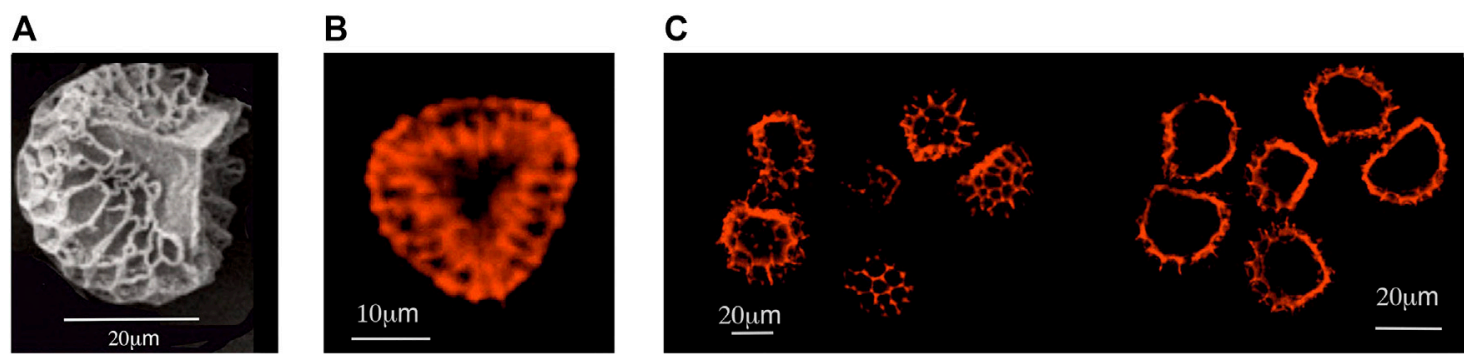

FIGURE 4 | (A), a SEM of spore of clubmoss. (B) a clubmoss exine doped with rhodamine B. To the (C), LSCM sections through functional particles of clubmoss doped with rhodamine $\mathrm{B}$, near to the surface, and in the bulk.

An example of this procedure is as follows: A $2 \mathrm{ml}$ solution $\left(0.1 \mathrm{~g} \mathrm{~L}^{-1}\right)$ of rhodamine B, Aldrich UK $98 \%$, (structure shown in Figure 2), in deionised water was added to the "column" and sucked through using a vacuum. The filtrate was isolated to determine the quantity of dye absorbed onto the "column". Further experiments were performed using different solvents as pre-treatments and dye type and solvent as listed in Table $\mathbf{1 .}$

The variety of solvents used for the same quantity of rhodamine $\mathrm{B}$ to be adsorbed, are shown in Table 2. The amounts adsorbed were determined from collection of the filtrate and removal of the solvent and weighing of the dye that remained un-adsorbed.

\section{Analysis of Microcapsules and Functional Particles}

The external and internal structures of empty microcapsules and filled particles were investigated by transmissive and reflective mode optical microscopy. They were inspected in both their dry forms, and in suspensions in various solvents, thereby enabling the examination of their quality and behaviour (coagulation) in solvents. SEM was used for the examination of microcapsule/ particle morphology; TEM $[18,19]$ provided information on the internal structures; and laser scanning confocal microscopy (LSCM) generated information on both internal structures and chemical natures. For example, Figure 4 shows LSCM sections [20] through functional particles of clubmoss doped with rhodamine B. Ultramicrotomy was employed where the samples were directly added to the Spurr resin and cut into $70 \mathrm{~nm}$ sections using a Leica Ultracut microtome. Further studies are to be made via EDS/EDX in our TEM experiments in order to determine the chemical compositions in the different regions of the samples.

\section{Magnetic Nanoparticles Inside Exine and Intine Capsules EICs}

In these studies, two methods were used to make exine capsules (EICs) that could be doped with nanomagnetic particles where the resulting magnetic spores (MSs) were responsive to magnetic fields. Such EICs were created by the direct synthesis of a magnetic material inside the exine microcapsules using the "micro-reactor" method, or by doping of magnetic nanoparticles into or onto sporopollenin, which is called the "doping" method. The methods utilized are reported in the Experimental section.

$\mathrm{Fe}_{3} \mathrm{O}_{4}$ nanoparticles were chosen as the magnetic material to be encapsulated into EICs, because compared to other magnetic materials, their production is relatively simple, and the reactants are relatively cheap and readily available. Also, Paunov et al. [21] reported a successful case for the encapsulation of $\mathrm{Fe}_{3} \mathrm{O}_{4}$ into exine microcapsules. In the experiment, $\mathrm{FeCl}_{2}$ and $\mathrm{FeCl}_{3}$ were firstly encapsulated into bleached exine and intine capsules (BEICs), and then the encapsulated BEICs were added to ammonia solution after quick washing with deionized water. Finally, the magnetic particles encapsulated BEICs (MS) were washed with water and dried in air.

\section{RESULTS AND DISCUSSION}

\section{The Encapsulation of Magnetic Particles}

The encapsulation of $\mathrm{Fe}_{3} \mathrm{O}_{4}$ makes the BEICs turn to a brown appearance, which may be caused by the reaction of ammonia and/or the presence of $\mathrm{Fe}_{2} \mathrm{O}_{3}$. There is no direct evidence to verify that the color change of the BEICs is caused by the introduction of an amino group, or if it is due to the formation of nanoparticles. However, an experiment was carried out in which BEICs were reacted with diaminoethane. The BEICs change from white to brown and the introduction of an amino group was considered the only likely reason for the color change. Furthermore, although $\mathrm{Fe}_{3} \mathrm{O}_{4}$ is a black material, it may be further oxidized to form $\mathrm{Fe}_{2} \mathrm{O}_{3}$, which is a brown-red material, after being encapsulated in BEICs.

Under light microscopy, apart from the colour, the dry MS1 particles had no perceived differences from the empty BEICs and no apparent damage was detected on this length scale. For magnetic nanoparticles obtained using a similar method, but in the absence of spores, aggregation can occur causing them to stick together to form aggregates that can be observed via microscopy. However, for the MS1 particles, there is no evidence of this using optical microscopy.

After suspension of the magnetic particles (MS1s) in toluene, some small dark red regions were observed under microscopy at 

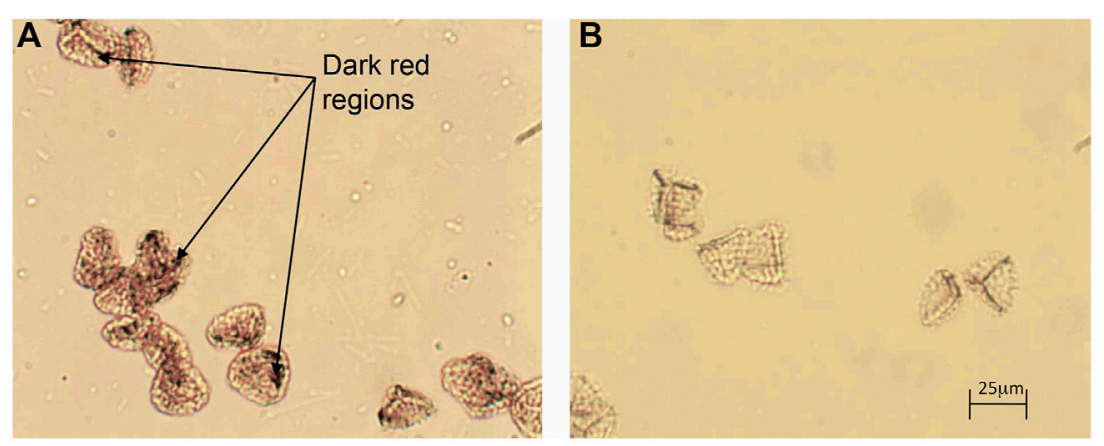

FIGURE 5 | (A) Photomicrograph of MS1s in toluene in transmissive mode with parallel polars (x 200), and (B) a photomicrograph of empty BEICs in toluene under parallel polars (x 200).

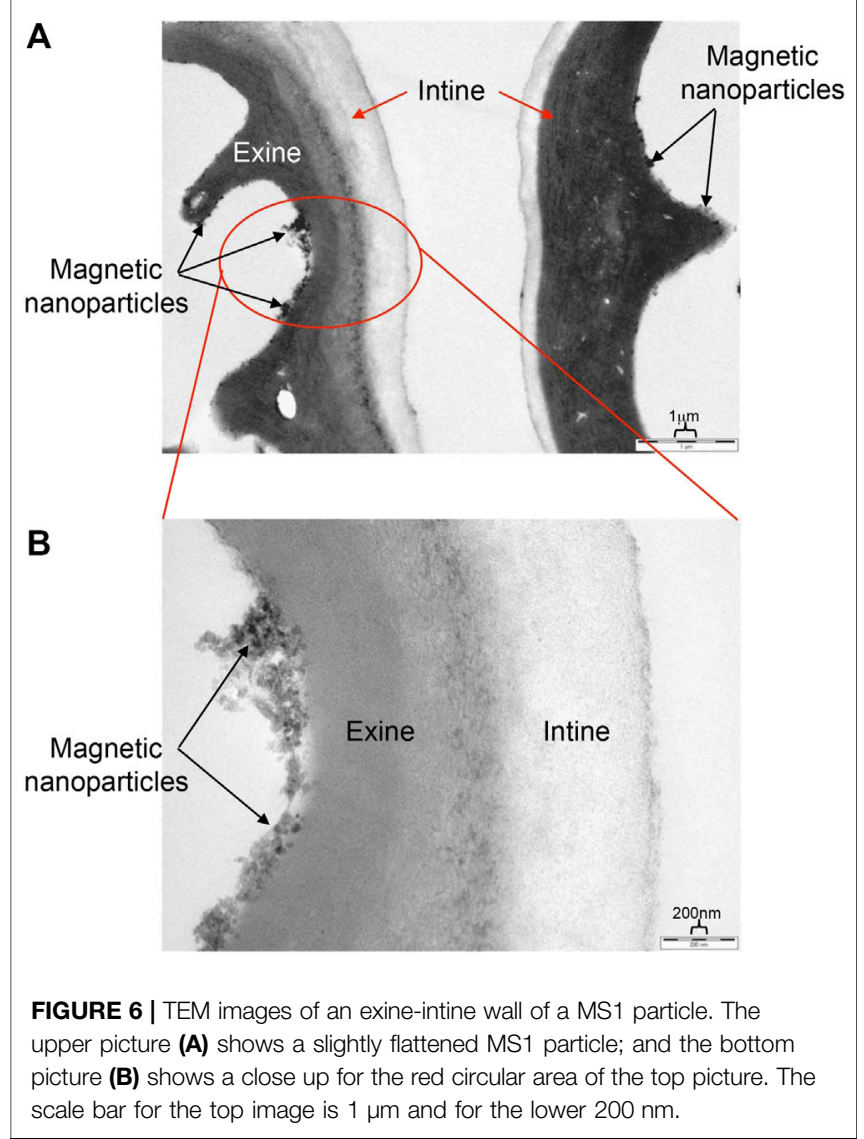

random locations on or in the exines, as shown in Figure 5A. Such observations do not occur with empty EICs, see Figure 5B, because due to the excess ammonia used in the reaction, the encapsulated $\mathrm{FeCl}_{2}$ and $\mathrm{FeCl}_{3}$ are mostly converted to $\mathrm{Fe}_{3} \mathrm{O}_{4}$. Therefore, the dark red regions may be caused by the presence of magnetic nanoparticles. However, it is hard to determine whether they are located inside or on the surface of the exine shells by optical microscopy alone.
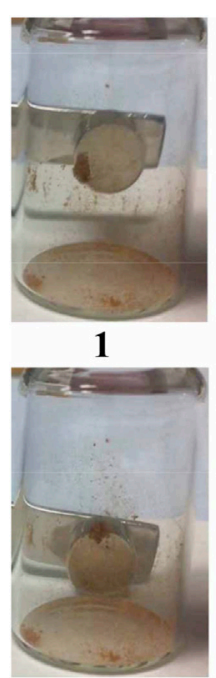

4

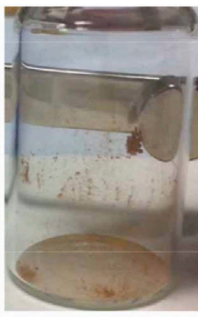

2

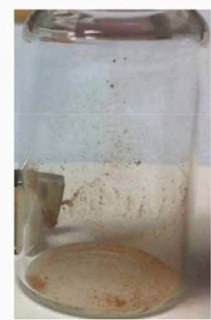

5

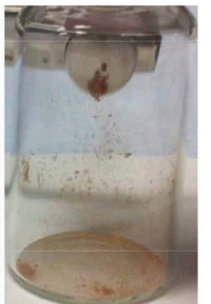

3

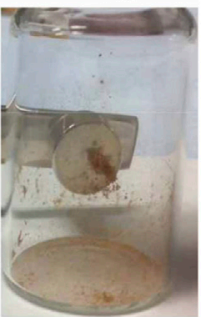

6
FIGURE 7 | Pictures taken from a video of MS1s moving by the application of a magnet field.

The purpose of the micro-reactor encapsulation method is to produce magnetic nanoparticles inside exine shells directly, bypassing the difficult process of filling the EICs with an insoluble material. As described in the method reported by Paunov et al., the starting reactants were initially encapsulated in the BEICs and then the filled BEICs were transferred into an ammonia solution for the formation of magnetic nanoparticles. It was expected that the ammonia solution would permeate the exine wall and react with the materials inside the exine microcapsules, resulting in the formation of magnetic nanoparticles. The particles are of varied shape as their formation occurs in an environment where there are local concentration gradients in the solvent, and surface versus bulk formation of crystal growth etc. Upon investigation by TEM, however, the $\mathrm{Fe}_{3} \mathrm{O}_{4}$ particles were only found to be present on the outer surfaces of the exine shells. Figure 6 shows the middle region of the cross-section of a MS1 


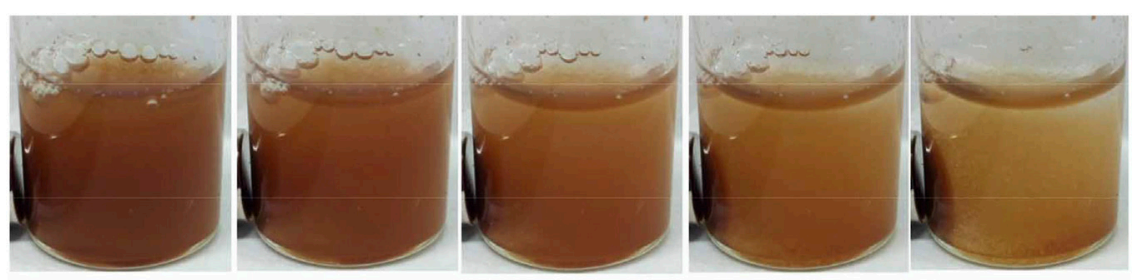

O s

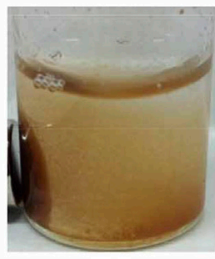

$35 \mathrm{~s}$
$5 \mathrm{~s}$

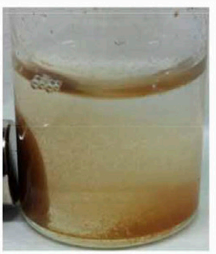

$60 \mathrm{~s}$
$10 \mathrm{~s}$

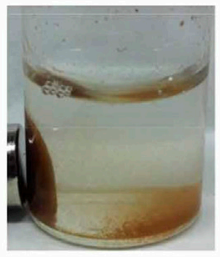

$100 \mathrm{~s}$
$15 \mathrm{~s}$

$25 \mathrm{~s}$

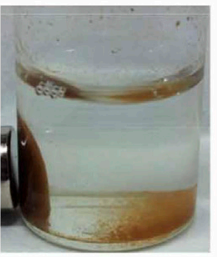

$180 \mathrm{~s}$

FIGURE 8 | Magnetic capsules suspended in water and subjected to a magnetic field as a function of time (seconds).

(EIC) and illustrates the presence of magnetic particles on the surface of exine shell only. All the TEM photographs of this sample show aggregated clusters and individual particles distributed randomly on the exine shell surface, covering a large proportion of the surface, with no particles permeating the intines.

There are three possible reasons why the nanoparticles are not encapsulated as follows: 1) The absence of magnetic nanoparticles may be due to the reactive materials exiting the spore prior to the reaction. However, this is unlikely as the target material is a salt and therefore would experience strong interactions with sporopollenin. 2) The reactants are not encapsulated in the exine microcapsules in the first place, but are deposited on the surface of exines because of the high affinity of sporopollenin to ionic salts. 3) The environment inside of the exines is not suitable for the formation of these types of particles, and so reaction takes place on the surface.

The magnetic nanoparticles that form on the surface of the exines are in the size range of $15-40 \mathrm{~nm}$ and possess irregular morphologies, as shown in Figure 6B. The nanoparticles must either interact strongly with, or be attached to, the surfaces of the exine walls because the exine capsules can respond and be moved by relatively weak applied magnetic fields. For example, Figure 7 shows dry MS1s being moved using a magnet placed near to the exterior of the sample vial. All of the exines in the sample of MS1 responded in a similar way to a weak magnetic field, indicating a relative uniformity in the distribution of the particles achieved in the encapsulation process. Upon movement of the magnet, the motions of some of the particles were hindered by friction and static charge on the inner surfaces of sample vial. This problem was resolved by suspending the sample in water or other solvents, such as acetone and ethanol.

The motions of magnetic capsules were also observed in water, starting with the time to collect near to the placed magnet placed at the wall of a vial. This simple experiment was started by firstly dispersing the capsules in water by shaking, as shown in Figure 8. The magnetic capsules moved quickly towards the magnet at the start, but as the cluster of capsules grew, the movement towards the magnet slowed. After $3 \mathrm{~min}$ in the presence of a magnet, most of the capsules sample gathered over a distance of $2 \mathrm{~cm}$ to the side of the vial. It should be noted that some of the capsules did not migrate to the wall of the vial and remained suspended in the water. It is possible that these EICs remained relatively empty on the reaction to form $\mathrm{Fe}_{3} \mathrm{O}_{4}$, or that the reaction was only partially complete. A further conclusion of the experiment is that magnetic microcapsules can be easily divided or isolated from nonmagnetic capsules in controlled separation processes, and that microcapusles can be used as chemically protected transport vehicles for infused materials to selected targets using magnetic fields. For example, specifically engineered lubricants may be transported to selected bearings.

A second empirical experiment to investigate the dynamic motion of magnetic capsules in an applied magnetic field was made via the rotation of the supermagnet adjacent to the external face of the vial. Firstly, the rotation of the external magnet had an effect on the clusters of the magnetic capsules. Upon rotation, slight movement of the spores can be observed and none of them appear to fall to the bottom of the vial. Individual capsules, however, showed controlled tumbling, but due the clustering of the microcapsules we cannot show the tumbling motion of individual capsules in bulk experiments. However, we can demonstrate the process via changes in the relative orientations of the microcapsules seen via optical microscopy. Further studies are already active in this area, and we have already examined the magnetophoretic behaviour of the capsules in applied fields. The results show that the microcapsules exhibit a spiraling motion, which seams to be dependent on capsule shape. The complexities of their behaviours are extensive in various solvents, which merit a separate article. 

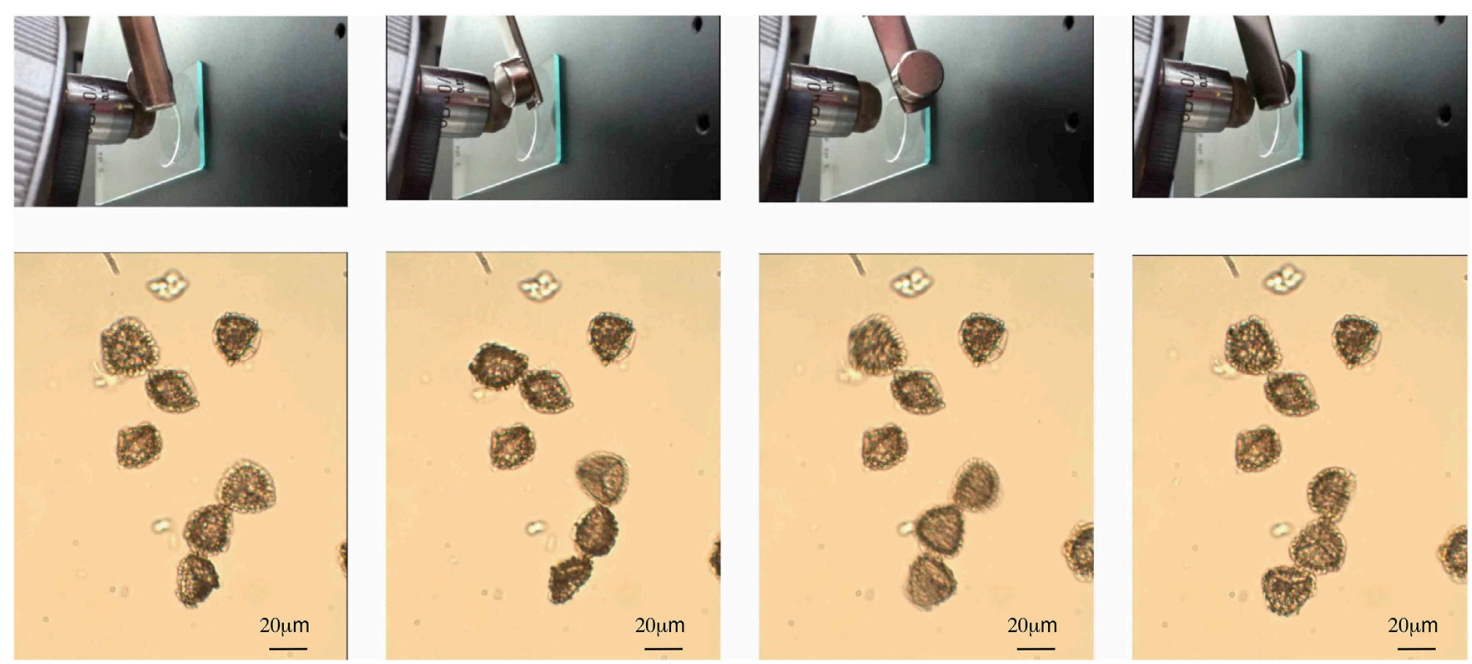

FIGURE 9 | Photomicrographs of magnetic capsules induced to rotate in water via an external magnetic field. The observations were made at room temperature using a polarizing microscope in transmission (x200).

In order to investigate the qualitative details of the rotation of magnetic capsules, an experiment was carried out under light microscopy with small quantities of MS1. Figure 9 shows the MS1s being rotated when the orientation of the magnetic field is changed. The rotation is very rapid and continuous, and yet the MS1s stay in the same position during rotation, and do not move toward the external magnet. There are three possibilities for this effect as follows; 1) the viscosity of the water mixture is too high for the exines to move in any linear distance with respect to the rotation of the magnet, 2) the magnet was too far away from the rotating MS1s and hence the field was too weak for linear motion, 3) the separation between the cover-slip and the slide was of comparable dimensions to the exines, and therefore the glass surfaces played a role, 4 ) the magnetic field induced turbulence in the motions of the exines due to non-uniform distributions of the magnetic nanoparticles on the surfaces of the exine shells, and 5) the flow of the particles in the water was in itself turbulent. Because of these possibilities, the rotations of the magnetic capsules were too hard to control. A solution to this issue may be to use multiple magnetic fields. In doing so, the combined strength and orientation of the magnetic field can be controlled to fix the motion of the magnetic capsule. Alternatively, the viscosity of the solvent is a possible reason why the particles did not migrate towards the magnet, however, it should be noted that the bulk sample didn't exhibit this problem.

\section{Doping of Magnetic Nanoparticles Onto Exine/Intine Capsules}

The aims of these studies were to create multifunctional microcapsules, which are doped with more than one functional material, such that they have more than one set of properties. Following on from the descriptions above, our target was to create coloured magnetic microcapsules that would respond to magnetic fields. In doing so we prepared MS capsules via different routes in creating "MS2" magnetic particles.

The second method used to produce magnetic exine/intine capsules (MS2EICs) was via directly doping magnetic nanoparticles onto bleached exines. This process is much simpler than that used for the MS1 system. The nanoparticles can be adhered to the surface of the exine shells using a variety of treatments, such as ultrasound and a "column" method. Each of the methods achieves similar results, but for the subsequent experiments all were completed using the magnetic capsules produced by the "column" method. This method involves packing bleached capsules (BEICs) in a pipette and compressing them with the use of a vacuum. A suspension of magnetic nanoparticles was added to the "column" and drawn through by reduced pressure until the column was dry. The magnetic nanoparticles used for MS2s were $\gamma-\mathrm{Fe}_{2} \mathrm{O}_{3}$, because it has a much smaller size $(\sim 10 \mathrm{~nm})$ than the nanoparticles that form within the MS1 system.

The visual appearances of the resulting coated MS2 particles were found to be similar to those of MS1s, as shown in Figure 10 left. Sweeping the sample with an external magnet left most of the non-magnetic particles isolated, thereby providing the possibility of mechanical removal via partition in the dry state, as shown in Figure 10 right. However, a small number of non-attached magnetic nanoparticles remained, as shown in the figure, whatever the method used to make the magnetic exines. The magnetic nanoparticles were expected to move more easily by an external magnet than MS2s, and therefore they should have been more easily isolated from the MS2 sample. However, entire removal was difficult without multiple sweeping with more than one magnet.

As well as isolation in dry conditions, it is also possible to isolate the MS2 capsules from non-magnetic exines in solvents under the application of a magnetic field, as shown in Figure 11. 
A

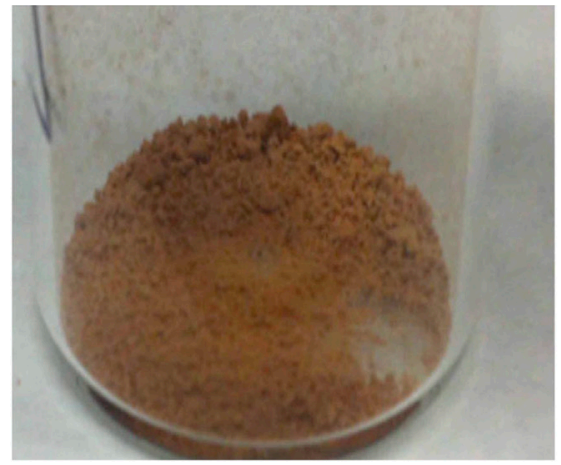

B

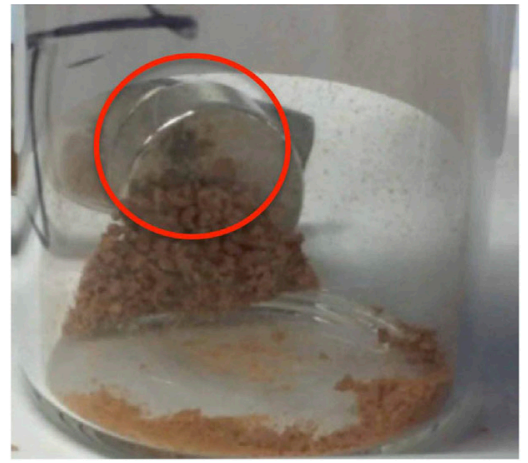

FIGURE 10 | Left (A) Photographs for the crude MS2 capsules, and Right (B) image showing the purification process occurring for MS2. The black powder highlighted by the red circle is thought to show an area composed of free magnetic nanoparticles, which are considered as impurities that can be partitioned via multiple sweeps of an external magnet(s).

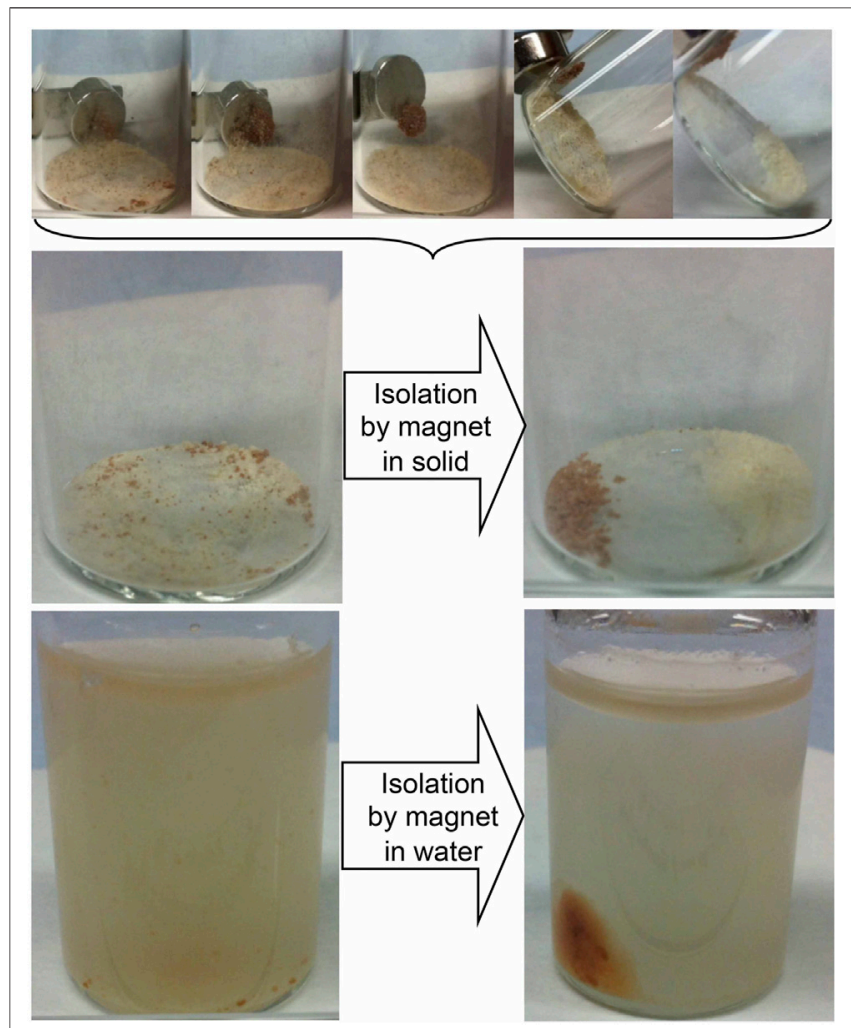

FIGURE 11 | Isolation of MS2 from empty bleached exines (BEIC) firstly using an external magnet in the dry (see top set of images, and the resultant separation, and subsequent Isolation of MS2 dispersed in water) again using an external magnet field.

In the bottom part of the figure the images show the MS2 capsules suspended in water, whereas the top of the figure shows the attraction of the magnetic entities under dry conditions. Partition occurs by dragging the magnetic particles clear of the clusters of non-magnetic exines. However, bulk separation is not so easy to achieve because of the clustering of various entities. In solution the suspended MS2 capsules are suspended more freely so that the magnetic particles can move more freely to cluster at the external magnet. Decanting or draining off of the remaining exines leaves the isolated and purified MS2 capsules adjacent to the external magnet and shown in the lower part of the figure in comparison to the central image that shows separation in the dry.

The TEM images of the MS2 capsules can demonstrate that the isolation and purification procedures can leave specimens free of magnetic nanoparticles associated with the exine/intine capsules (EICs), and in the images almost all associated magnetic particles were located on the outer surface of the exine shells, as shown in Figure 12. Figure 12A shows a large area of a capsule wall, which shows aggregates/clusters of nanoparticles on the surfaces, with none located within the walls. The blown-up image (Figure 12B) shows the clusters are not surrounded with smaller domains of magnetic particles, unlike the nanoparticles found for MS1 capsules, and that they only stay on the surfaces of the exine walls. The reason for this is difficult to rationalise, for instance, it may have been caused in the preparation of the TEM sample or at any step in the procedure to prepare MS2s. Nevertheless, the TEM images for the MS1 capsules were prepared by the same method as for MS2s, but it uses instead a completely different approach to coating nanoparticles onto the EICs.

Thus, as the TEM images for the MS1 capsules show that some nanoparticles can also be doped into the exine wall, the preparation of TEM samples is probably the more likely reason for the appearance of nanoparticles in the exine walls. This is because the sizes of the particles are obviously larger than the nanotubes within the exine walls. Thus the nanoparticles were formed on the surfaces of the exine walls rather than in it. Therefore, when the nanoparticles appeared partially within or overlapped on the exine walls, it is more likely to have been caused by cut/slide action in the TEM sample preparation process. Nevertheless, in spite of the difference in the purities and the sizes of the nanoparticle clusters, the behaviour of MS2 and MS1 capsules are similar in their responses to magnetic fields.

Although some of the nanoparticles that attach to the bleached exine/intine microcapsules (BEICs) in the magnetic spores type 2 (MS2s) do so as aggregates not individual particles, 


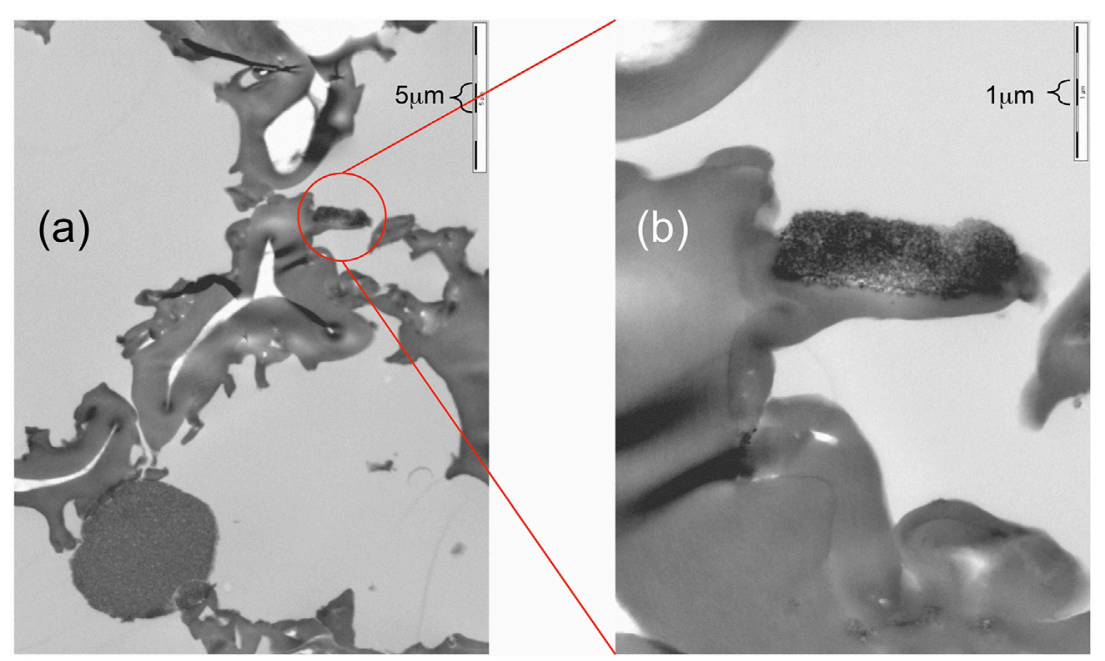

FIGURE 12 | TEM images of a MS2 microcapsule showing nanoparticles on the surfaces of the exine walls. Scale (A) $5 \mu \mathrm{m}$ and (B) $1 \mu \mathrm{m}$.

this does not appear to have an apparent effect on the response of MS2s to the rotation of the magnetic field. They can still rotate as MS1s did under microscopy. However, the MS2s move easier toward the magnet than the comparative MS1s, but as a consequence the doped exines do not necessarily remain in the same local position when they are rotated.

Compared with MS1s, the preparation process of MS2s capsules is relatively straightforward. It is possible to avoid altering the chemical structure of sporopollenin and the bio-content if still present (e.g. residue of intine material and protoplast) by materials such as ammonia, achieving similar effects as for MS1s. MS2s, however, can possesses relatively lower purities, which is mainly as a result of free magnetic nanoparticles mixed within the product. It should be noted, however, that in order to effectively coat the exines with nanoparticles, a treatment such as ultrasound is usually required, which in turn limits the contamination from free nanoparticles to a certain degree (see Experimental section).

Furthermore, the method of doping magnetic nanoparticles onto exine shells may also be used to dope magnetic nanoparticles onto other micro-scale objects, such as cells, using a similar procedure. After doping of magnetic nanoparticles, the microobject might be rotated and moved by a magnetic field, allowing observations from all sides for the object.

\section{Multifunctional Exine and Intine Microcapsules}

In the above, exine and intine capsules (EICs) have only been filled with a single material that possesses only one function, in this case magnetic. However, it is possible to encapsulate more than one functional material inside EICs, thereby producing multi-functional microcapsules. If multiple materials are to be simultaneously encapsulated inside EICs there are several considerations to make, for example, the materials should be carefully selected, so as to produce optimum physical properties

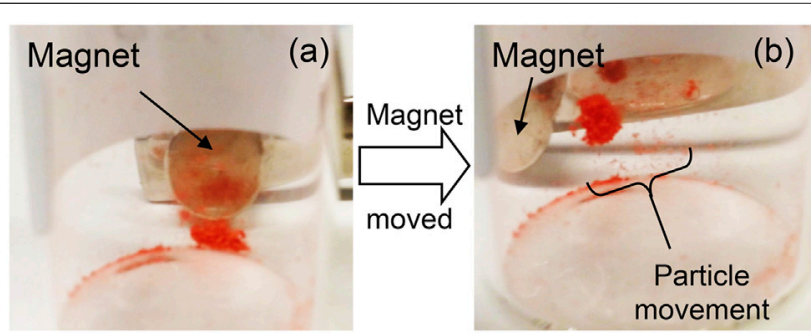

FIGURE 13 | (A) The dyed magnetic capsules and external magnetic before movement, and $(\mathbf{B})$ the particle movement caused as the position of the magnet was altered.

and to avoid any undesirable chemical side reactions between the encapsulated materials and/or EICs. It would be desirable to encapsulate materials in the minimum number of steps. Otherwise, a few problems may occur en route, such as the leaking of encapsulated material caused by the next encapsulation steps and loss of product by transferring steps. For instance, experience has shown that a dye mixture may be encapsulated, but differential dye leakage can occur when the functionalised microcapsules are suspended in various solvents. Thus in the following study we chose the use of coloured and magnetic materials to demonstrate the possibility of producing two-component functional microcapsules. The magnetic material was based upon $\gamma$ $\mathrm{Fe}_{2} \mathrm{O}_{3}$, the dye on fluorescent rhodamine $\mathrm{B}$, and the microcapsules generated in process to form MS2s. In this way the magnetic nanoparticles remain on/in the outer shells of the exines, and the dye fills the empty interior, i.e. they remain separated and do not exhibit cross-problems of filling.

In order to produce such bifunctionalized microcapsules, rhodamine B was added to MS2s (magnetic spores described above, and in detail in the Experimental Section using a 

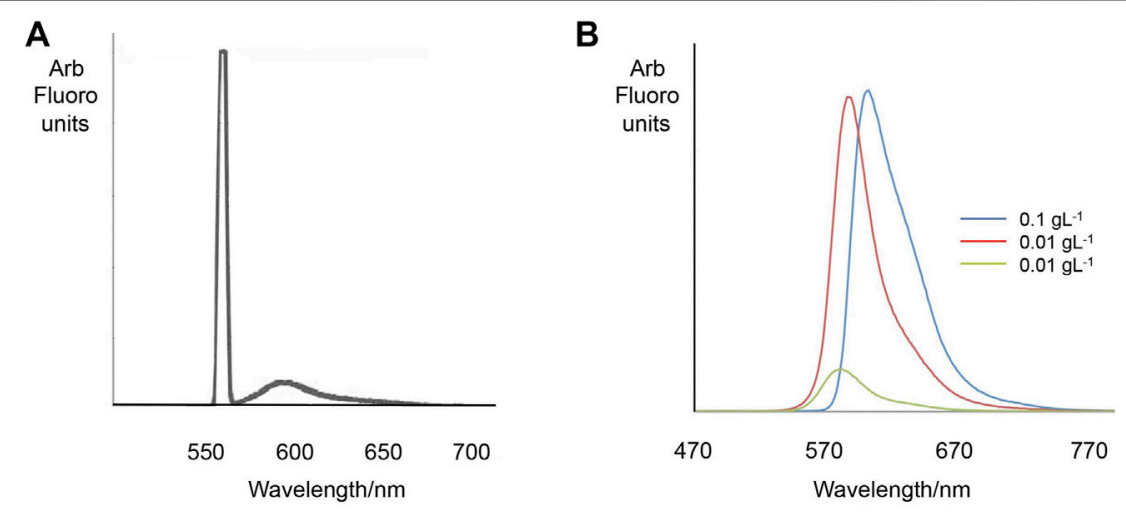

FIGURE 14 | Fluorescence emission spectra of rhodamine dye doped MS2 particles (A). Excitation wavelength: 554 nm. The peak emission wavelength of RB in aqueous solution (range 550-700 nm) (B)

microcolumn). The magnetic EICs (MS2s) that were used to produce the dyed capsules were light brown in colour, but after encapsulation of the dye the brown colour was no longer observed and the bright pink of the dye could be clearly seen, thereby producing bright pink microcapsules.

The magnetic response of the dye filled MS2s was found to be very similar to that of the un-dyed parent capsules, i.e. in the presence of a magnet, both dyed and un-dyed capsules move towards the magnet in the same direction with similar speeds, see Figure 13, suggesting that the additional encapsulation of rhodamine $\mathrm{B}$ does not alter the observed magnetic properties of the microcapsules. The dyed capsules were also expected to fluoresce due to the presence of the fluorochromic rhodamine $B$ and sporopollenin exine. However, the emission and absorption profiles for both were not as pronounced, possibly due to the presence of the magnetic nanoparticles. Nevertheless, the presence of two peaks were observed in the fluorescence emission spectrum as shown in Figure 14; a sharp peak at 550-560 probably from excitation light beam (554 nm), and another broad peak at 560-650 from the encapsulated rhodamine B. However, the signal from the bleached exines (BEICs) was not observed, possibly due it being too weak to be detected in comparison with the other obvious peaks. However, the intensity of the peak for the rhodamine $B$ indicates that a substantial quantity had been adsorbed into the exine shell. But as shown in Figure 2, the dye and other materials also appear stable over a period of at least 10 years, indicating the degree of protection provided by the sporopollenin outer shell.

TABLE 3 | Emission spectra of aqueous solutions of rhodamine $B$ at different concentrations. Excitation wavelength $365 \mathrm{~nm}$.

Concentration of RB solution $\left(\mathrm{g} \mathrm{L}^{-1}\right)$

0.1

0.01

0.001

\section{Peak} emission wavelength $(\mathrm{nm})$

601

586

579
Subsequently, we examined the variation in the rhodamine B emissions that occur over the region $550-670 \mathrm{~nm}$. We found that as the concentration of the RB solution used to fill BEICs is increased (see Table 3), the peak emission wavelength in the range $550-700 \mathrm{~nm}$ (corresponding to the encapsulated $\mathrm{RB}$ ) shifts to longer wavelengths. However, this observation is not particular to encapsulated $\mathrm{RB}$, but also occurs in aqueous solutions (Figure 14B). This observation is thought to be caused by the fluorescence re-absorption between the rhodamine B molecules. This shift is therefore not solely related to the presence of BEICs. Furthermore, the proportion of BEICs' emission quenched by encapsulated $\mathrm{RB}$ increases as the concentration of the RB solution used during encapsulation was increased. This suggests more energy should be absorbed by or transferred to encapsulated $\mathrm{RB}$ from the BEICs. Therefore, the interaction between BEICs and $\mathrm{RB}$ should shift the RB peaks to higher frequency. However, the fact that this does not happen supports the argument that the presence of BEICs has either a very limited, or has no effect on the fluorescent behaviour of RB.

We further examined the fluorescence of the BEICs in comparison to related dyed specimens using spectrofluorometry in a front-face excitation geometry. Two batches, 1 and 2, of BEICs of slightly different colour were prepared using the same method. Batch 1 was slightly whiter than 2, although the difference was very small and did not affect the performances of the samples. Experiments on the batches were analyzed using spectrofluorometry and the results are shown in Figure 15 ( $\mathrm{a}$ and b). Samples based on BEIC batches one and two were found to exhibit two peaks in their spectra; one between $380-500 \mathrm{~nm}$ and the other at $729 \mathrm{~nm}$. The peaks between $380-500 \mathrm{~nm}$ were due to the autofluorescence of sporopollenin, as well as fluorescence from other components of the spores, such as the intine and protoplast [22,23]. Conversely, the peaks at $729 \mathrm{~nm}$ were probably due to second order diffraction and are caused by the excitation light. Second order diffraction usually occurs from strongly scattering surfaces, such as from the samples prepared for this study. These peaks can be eliminated if a $350 \mathrm{~nm}$ long-pass filter is situated between the 

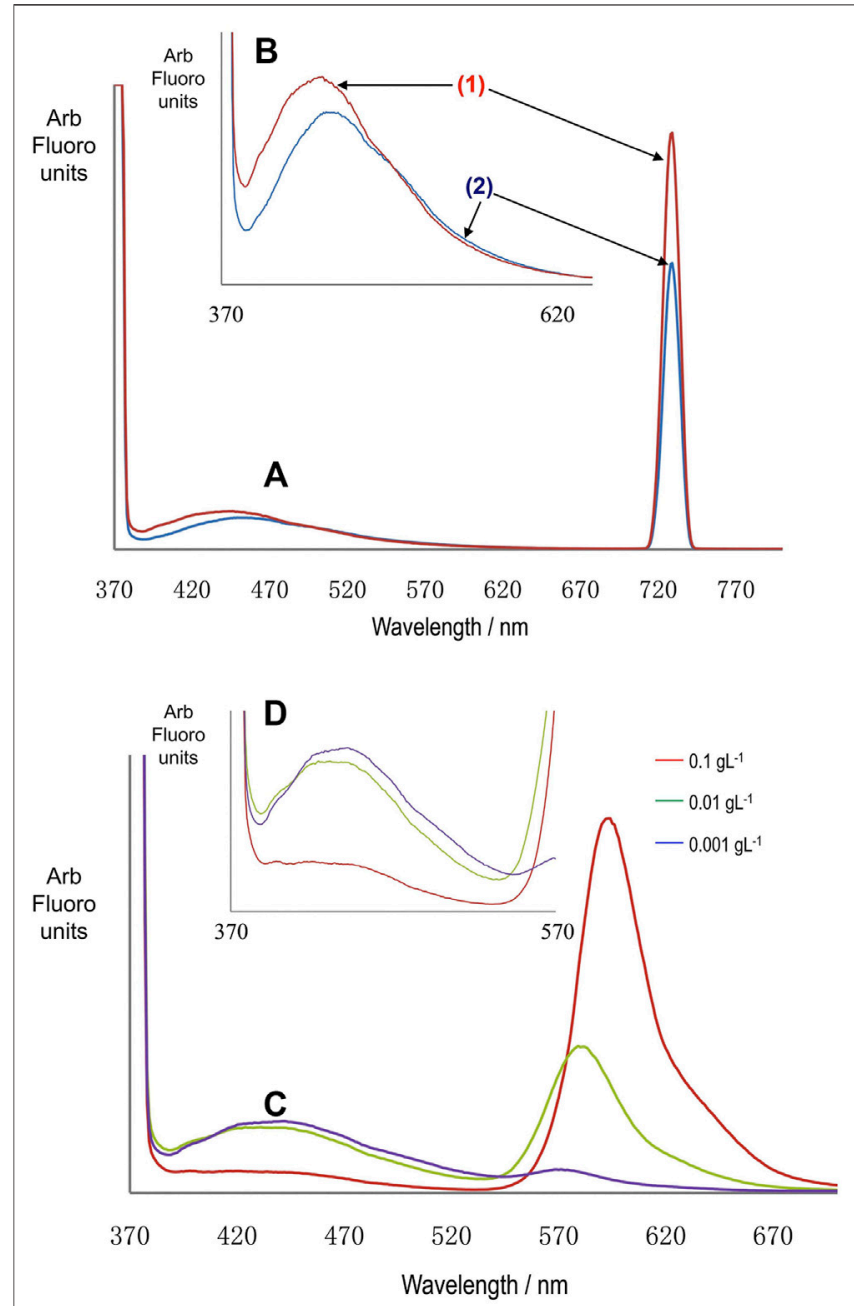

FIGURE 15 | Graphs (A,B) are emission spectra for BEICs from two different batches [(1) and (2)] of spores. Spectrum (B) is an expansion of the 370-650 nm range of the spectrum (a). The peak emission wavelength for (2) is $444 \mathrm{~nm}$ and for (1) $449 \mathrm{~nm}$. Graphs (C,D) are spectra of the BEICs treated with aqueous solutions of rhodamine $B$ at the concentrations shown. Graph (D) is an expansion of the region $370-570 \mathrm{~nm}$ of graph (C). Excitation wavelength is $365 \mathrm{~nm}$ for all of the spectra.

sample and the detector. However, as a long-pass filter was not used in this work, peaks situated at $\sim 729 \mathrm{~nm}$ were excluded from analysis when a $365 \mathrm{~nm}$ excitation beam was used. Furthermore, it should be noted that although one and two appeared visually different, their emission profiles as shown in the spectra were broadly similar. The BEIC batches were filled using various concentrations of rhodamine $\mathrm{B}$ in water $(0.1,0.01$, and $0.001 \mathrm{~g} \mathrm{~L}^{-1}$ ) and their fluorescence emission spectra analyzed, as shown in Figures 15C,D for dyed batch 1. Peaks attributed to autofluorescence of BEICs still remain in the spectra (between $370-530 \mathrm{~nm}$ ), but the peak emission wavelength of these bands appeared to shift to shorter wavelengths at higher rhodamine B concentrations. These are very broad bands, so assigning maxima is difficult, meaning that very small shifts in peak emission wavelengths may be insignificant. The peak area of BEICs' emission decreases relative to that of rhodamine $B$ as the concentration of rhodamine B is increased. Therefore, the emission from the BEICs appears quenched in the presence of the dye.

Other dyes have also been deployed in preparing coloured exine/intine microcapsules and their magnetic equivalents. The dyes investigated included naphthol blue-black, methylene blue hydrate, safranin $\mathrm{O}$, purple anthraquinone, and thiazole yellow $\mathrm{G}$, which gave us a range of dyes from ionic and water-soluble to non-ionic and non-water soluble. Mixtures of magnetic and nonmagnetic dye systems were also prepared, as for example a mixture of rhodamine B magnetic and thiazole yellow G nonmagnetic capsules. For dyes adsorbed into BEICs the colour depends on the dye and on the microcapsules for various powder specimens, and when dyed BEICs are mixed the colour averages depending on the relative proportions of the components. For a mixture of dyed magnetic and dyed non-magnetic capsules, the application of an applied magnetic field can be used to separate the mixture with the magnetic capsules being isolated by movement towards an external magnet leaving the non-magnetic capsules stranded. This demonstrates that these systems may be useful as molecular transport vehicles for delivering a material to a selected target. For certain applications, selected coloured dyes may be required, for instance in printing or paints etc. Such coloured and protected particles may be created by either dye mixing prior to adsorption, or by mixing dyes internally within the microcapsules.

\section{SUMMARY}

In these studies we demonstrate that we can prepare relatively large quantities ( $70 \mathrm{~g})$ of untainted emptied spores of clubmoss (Lycopodium clavatum) to give robust microcapsules [24] of defined size, which can be doped onto their surfaces with magnetic materials or filled with a variety of ionic and nonionic dyes, singularly or together. We also show that the resulting coloured particles of choice can physically respond to the application of an externally applied magnetic field both in terms of linear and rotational fields. We also demonstrate that magnetic microcapsules can be separated from non-magnetic entities both in a dry state and suspended in a solvent. We have worked extensively with the laser dye rhodamine B because of its various spectral properties and conversely because of its relative, chemical instability which could be tested under various conditions. When encapsulated in the emptied spores, over a 10 -year period rhodamine $\mathrm{B}$ has shown no degradation under dry conditions or in a suspended state in acetone.

Thus, these studies show that we are in a position to further develop a branch of "Separations Science" where functional particles of different type may be separated depending on external fields. However, what we have not explicitly demonstrated in these studies is that "material-passengers" within the exines may be recovered. This is usually possible under mild conditions, meaning that passengers may be protected over long periods of time and yet still be recovered. 
Furthermore, these studies also show that plant spores can be used to create multifunctionalized complex microcapsules, which when doped with nanomagnetic substances can be delivered to various sites under an applied magnetic field, and then emptied.

\section{DATA AVAILABILITY STATEMENT}

The raw data supporting the conclusion of this article will be made available by the authors, without undue reservation.

\section{AUTHOR CONTRIBUTIONS}

All authors listed have made a substantial, direct, and intellectual contribution to the work and approved it for publication.

\section{REFERENCES}

1. Strohl WR, Larkin JM, Good BH, Chapman RL. Isolation of Sporopollenin from Four Myxobacteria. Can J Microbiol (1977) 23:1080-3. doi:10.1139/ m77-162

2. Gooday GW, Fawcett P, Green D, Shaw G. The Formation of Fungal Sporopollenin in the Zygospore Wall of Mucor Mucedo: a Role for the Sexual Carotenogenesis in the Mucorales. J Gen Microbiol (1973) 74:233-9. doi:10.1099/00221287-74-2-233

3. Kim SS, Douglas CJ. Sporopollenin Monomer Biosynthesis in Arabidopsis. J Plant Biol (2013) 56:1-6. doi:10.1007/s12374-012-0385-3

4. Chauhan AK, Varma A. Textbook of Molecular Biotechnology. I.K. New Delhi: International Publishing House Pvt. Ltd (2009). p. 64-5. Ch. 1.

5. Brooks J, Shaw G. Sporopollenin: A Review of its Chemistry, Palaeochemistry and Geochemistry. Grana (1978) 17:91-7. doi:10.1080/00173137809428858

6. Ariizumi T, Toriyama K. Genetic Regulation of Sporopollenin Synthesis and Pollen Exine Development. Annu Rev Plant Biol (2011) 62:437-60. doi:10. 1146/annurev-arplant-042809-112312

7. Sutton MQ, Sobolik KD, Gardner JK. Paleonutrition. Tucson: The University of Arizona Press (2010). p. 109-10. Ch. 4.

8. Shaw G, Harborne JB. Phytochemical Phylogeny. London and New York: Academic Press (1997). p. 31-5. Ch. 3.

9. Wittrup A, Zhang S-H, Svensson KJ, Kucharzewska P, Johansson MC, Morgelin M, et al. Magnetic Nanoparticle-Based Isolation of Endocytic Vesicles Reveals a Role of the Heat Shock Protein GRP75 in Macromolecular Delivery. Proc Natl Acad Sci (2010) 107:13342-7. doi:10.1073/pnas.1002622107

10. Gong X, Peng S, Wen W, Sheng P, Li W. Design and Fabrication of Magnetically Functionalized Core/Shell Microspheres for Smart Drug Delivery. Adv Funct Mater (2009) 19:292-7. doi:10.1002/adfm.200801315

11. Hwang YK, Choi JN, Cho JH, Kwon H, Huh S. Fe 3 O 4 -NanoparticleEmbedded Multifunctional Hollow Mesoporous Silica Capsules. Eur J Inorg Chem (2012) 2012:3379-83. doi:10.1002/ejic.201200385

12. Gouin S. Microencapsulation. Trends Food Sci Technology (2004) 15:330-47. doi:10.1016/j.tifs.2003.10.005

13. Sarkar TR, Irudayaraj J. Carboxyl-coated Magnetic Nanoparticles for mRNA Isolation and Extraction of Supercoiled Plasmid DNA. Anal Biochem (2008) 379:130-2. doi:10.1016/j.ab.2008.04.016

14. Lorch M, Thomasson MJ, Diego-Taboada A, Barrier S, Atkin SL, Mackenzie G, et al. MRI Contrast Agent Delivery Using Spore Capsules: Controlled Release in Blood Plasma. Chem Commun (2009) 42:6442-4. doi:10.1039/b909551a

15. Zetzsche F, Kälin O. Untersuchungen über die Membran der Sporen und Pollen V. 4. Zur Autoxydation der Sporopollenine. Hca (1931) 14:517-9. doi:10.1002/hlca.19310140151

\section{FUNDING}

WC and CB were self-funded, SC was a University of York Senior Research Fellow, and JG is Emeritus Professor of Materials Chemistry.

\section{ACKNOWLEDGMENTS}

We acknowledge the support of Isabel Saez, Katrina Bakker, Emily Bevis, Richard J. Mandle and John Moore and for their many useful discussions. We are also indebted to Grahame Mackenzie of the University of Hull who inspired us to begin our research into the use of plant spores as a source of natural microcapsules.

16. Diego-Taboada A, Maillet L, Banoub JH, Lorch M, Rigby AS, Boa AN, et al. Protein Free Microcapsules Obtained from Plant Spores as a Model for Drug Delivery: Ibuprofen Encapsulation, Release and Taste Masking. J Mater Chem B (2013) 1:707-13. doi:10.1039/C2TB00228K

17. Atkin S, Beckett S, Mackenzie G. Inventors, University of Hull Assignee. Whitened Exine Shells. WO2010004334 Patent (2008).

18. Weakley BS. Beginner's Handbook in Biological Transmission Electron Microscopy. Edinburgh: Churchill Livingstone Press (1981).

19. Hayat A. Principles and Techniques of Electron Microscopy: Biological Applications. Cambridge: CRC Press (1989).

20. O'Connor DJ, Iacopino D, Healy DA, O'Sullivan D, Sodeau JR. The Intrinsic Fluorescence Spectra of Selected Pollen and Fungal Spores. Atmos Environ (2011) 45:6451-8. doi:10.1016/j.atmosenv.2011.07.044

21. Paunov VN, Mackenzie G, Stoyanov SD. Sporopollenin Micro-reactors for InSitu Preparation, Encapsulation and Targeted Delivery of Active Componentsin-Situ Preparation, Encapsulation and Targeted Delivery of Active Components. J Mater Chem (2007) 17:609-12. doi:10.1039/B615865J

22. Lin CP, Kim C, Smith SO, Neiman AM. A Highly Redundant Gene Network Controls Assembly of the Outer Spore wall in S. cerevisiae. PLoS Genet (2013) 9:e1003700. doi:10.1371/journal.pgen.1003700

23. Audran JC, Willemse MTM. Wall Development and its Autofluorescence of Sterile and fertileVicia Faba L. Pollen. Protoplasma (1982) 110:106-11. doi:10. $1007 / \mathrm{bf} 01281536$

24. Mackenzie G, Boa AN, Diego-Taboada A, Atkin SL, Sathyapalan T. Sporopollenin, the Least Known yet Toughest Natural Biopolymer. Front Mat (2015) 2. doi:10.3389/fmats.2015.00066

Conflict of Interest: The authors declare that the research was conducted in the absence of any commercial or financial relationships that could be construed as a potential conflict of interest.

Publisher's Note: All claims expressed in this article are solely those of the authors and do not necessarily represent those of their affiliated organizations, or those of the publisher, the editors and the reviewers. Any product that may be evaluated in this article, or claim that may be made by its manufacturer, is not guaranteed or endorsed by the publisher.

Copyright $\odot 2022$ Cai, Bradbury, Cowling and Goodby. This is an open-access article distributed under the terms of the Creative Commons Attribution License (CC BY). The use, distribution or reproduction in other forums is permitted, provided the original author(s) and the copyright owner(s) are credited and that the original publication in this journal is cited, in accordance with accepted academic practice. No use, distribution or reproduction is permitted which does not comply with these terms. 